Estudios de la Paz y el Conflicto

Revista Latinoamericana

IUDPAS-UNAH

Número Especial 1, pp. 53-65

ISSN-e: 2707-8922 / ISSN: 2707-8914

DOI: $10.5377 /$ rlpc.v0i0.9503

Anual 2019

\title{
LA PSICOLOGÍA COMO DISCIPLINA EN TIEMPOS DE POST ACUERDO DE PAZ EN COLOMBIA
}

\author{
PSYCHOLOGY AS FIELD IN TIMES OF TRANSITION IN COLOMBIA
}

\author{
Diana Marcela Agudelo Ortiz \\ Universidad Externado de Colombia \\ diana.agudelo@uexternado.edu.co
}

Cómo citar / citation

Agudelo, DM. (2019). "La psicología como disciplina en tiempos de post acuerdo de paz en Colombia", Estudios de la Paz y el Conflicto, Revista Latinoamericana, Número

Especial 1, 53-65. DOI: 10.5377/rlpc.v0i0.9503

\begin{abstract}
Resumen
La psicología como disciplina social y humana, entendida como una plataforma amplia de saberes y prácticas para la comprensión e intervención de los procesos subjetivos cumple un rol fundamental para la construcción de paz en Colombia. La atención a víctimas y los procesos de desmovilización, de reintegración y de reincorporación, son apenas algunos de los desafíos en los que se demanda la presencia de profesionales capaces de acompañar en la transición del dolor a una sociedad que no ha tenido tiempo para dar sentido a una guerra de más de 60 años. En ese sentido, la formación de profesionales en un campo con creciente demanda como el de la psicología, implica problematizar su relación con la búsqueda de justicia y la profundización en las perspectivas con las cuales se articulan vías para la construcción de paz y la reconstrucción del tejido social en Colombia.
\end{abstract}

\section{Palabras claves}

Psicología; Educación para la paz; Acuerdos de paz Colombia.

\begin{abstract}
Psychology as a human and social field plays a crucial role for peace building processes in Colombia as it implies a wide platform of knowledges and practices addressing to the comprehension and intervention of subjective processes. Victims attention and services along with DDR processes of different armed groups are some of the challenges for the professionals involved in the transition from pain and protracted violence of a society that hasn't had much time to provide meaning to a war lasting more than 60 years. Psychology
\end{abstract}


is one of the most popular careers in Colombia, thus professional education in this field requires a lot of work in terms of debate and problematization of its own links with justice pursuit and on how it contributes to peace building and social fabric reconstruction.

\section{Palabras clave}

Psychology; Education for peace; Peace agreements Colombia.

\section{INTRODUCCIÓN}

Esta propuesta tiene como objetivo reflexionar sobre la formación y el ejercicio de la psicología en Colombia, en el marco de las dinámicas del presente, marcadas por el proceso de post acuerdo con las Fuerzas Armadas Revolucionarias de Colombia (en adelante, FARC), y más ampliamente por todos los escenarios que éste ha abierto, como el reconocimiento de las víctimas, la apertura de espacios institucionales del Estado para trabajar en el tema, y particularmente la posibilidad para la población civil de dar sentido a los acontecimientos de los últimos años en el país.

La reflexión se propone desde un proceso de formación de investigadores en ciencias sociales y humanas con una apuesta interdisciplinar, en donde participa la psicología y del que la autora ha formado parte como estudiante y ahora como docente investigadora. Dicha propuesta retoma la necesidad de convocar la producción de conocimiento desde los sujetos (Zemelman, 2011) y las realidades que los atraviesan más allá de las disciplinas, pero también problematiza la formación profesionalizante especialmente en los campos de intervención que requieren renovadas lecturas del contexto socio histórico y político de un país como Colombia, en el cual, la academia hasta hace muy pocos años está haciendo la transición de los estudios de las violencias hacia la construcción de paz en el marco de las desmovilizaciones colectivas de los grupos paramilitares entre 2003 y 2006, y del acuerdo de paz de La Habana del 2016, firmado entre el gobierno de Colombia y la guerrilla más antigua del continente, las FARC-EP.

Así, se introduce el problema de la memoria como agente educativo (Arias, 2015), la ausente discusión sobre los saberes de la guerra (Sánchez, 2017) y la recurrente distancia que la población estudiantil argumenta en relación con las experiencias vividas incluso por sus propios familiares; y la relación de todo esto con la postura y participación ciudadana en los procesos como el plebiscito y la polarización frente a los procesos de justicia transicional que vivimos hoy tras el acuerdo de paz.

Se propone que el papel del trabajo con narrativas autobiográficas permite abrir espacios pedagógicos dentro y fuera del aula para la desnaturalización del conflicto armado y para develar la profunda raíz que tienen las dinámicas de guerra en el espectro subjetivo del colombiano, con el fin de construir puentes y estrategias hacia la reconciliación y la restitución de los vínculos y del tejido social.

Se reflexiona además sobre el riesgo de la instrumentalización del saber psicológico en los escenarios de atención a la población que ha sido víctima de la violencia, pero también de los excombatientes, argumentando la necesidad de una claridad política por la defensade la vida, la dignidad y los derechos humanos de los individuos, colectivos y territorios con quienes trabajamos. 
Finalmente se explora la precariedad laboral con la que los profesionales que ejercen la labor psicosocial en el país deben asumir su trabajo de jalonar los procesos de reconstrucción del tejido social, de creación de confianza y de construcción de paz, muchas veces en medio de la inestabilidad laboral de las mismas instituciones que los contratan, en un país que en el que el Registro Único de Víctimas ${ }^{1}$ reporta al 1 de enero de 2020 un total de 8'553.416 personas y 10'608.112 de hechos victimizantes; mientras que por su parte la Agencia para la Reincorporación y Normalización reporta un histórico de 74.855 personas desmovilizadas de grupos armados al margen de la ley en Colombia, entre los años 2001 y 2019². Estos datos nos ayudan a dimensionar la magnitud del desafío que tenemos en nuestras manos.

\section{EL LUGAR DE ENUNCIACIÓN}

En mi experiencia docente con estudiantes de psicología me enfrento constantemente con la dificultad de superar la obviedad que nociones como la de subjetividad social suponen. Y es que parece que todos estamos de acuerdo en decir que los sujetos son sociales, pero cuando profundizamos en lo que aquello quiere decir no resulta tan claro ni evidente, pues no se dice nada diferente a que los vínculos, las relaciones sociales y los contextos son importantes, algo así como "lo social" es muy importante para la comprensión de lo psicológico, cuestión que dice todo y nada al mismo tiempo. Prueba de ello es la dificultad que enfrentan para comprender que su subjetividad está fuertemente atravesada por el conflicto armado interno, aunque este haya sido visto solo por televisión.

Esto resulta muy revelador para una Facultad que está fundamentada en la formación de investigadores sociales en Colombia. Y es que, como tal, la Facultad de Ciencias Sociales y Humanas de la que hablamos (en adelante, FCSH), está planteada de tal manera que se estimula la producción de conocimiento en todos sus espacios y desde el primer semestre. Además de la investigación, el pilar complementario es una perspectiva interdisciplinar que favorezca dicha producción de conocimiento más allá de las barreras disciplinares. Esta es la Facultad donde estudié y en donde hoy en día además de investigación social en campos diversos, enseño psicología.

La FCSH busca formar investigadores sociales capaces de comprender, problematizar, intervenir y transformar las realidades de las distintas poblaciones, instituciones y organizaciones con las que trabajan, de acuerdo a las dinámicas territoriales y al contexto histórico social donde se encuentran insertas. De acuerdo con esto y con los 17 años que tiene de abierta la Facultad, se plantean algunas de las preguntas que han ido emergiendo como resultado y escenario de tensión para una disciplina como la psicología, que investiga, produce conocimiento y se mueve en favor de la defensa de la vida de los sujetos con los que trabaja, tanto en lo que tiene que ver con el proceso de formación de estudiantes de pregrado como del quehacer mismo de los profesionales en psicología.

\footnotetext{
${ }^{1}$ El Registro Único de Víctimas es una base de datos a nivel nacional que actualiza permanentemente las cifras de la Unidad para la atención y reparación integral a las víctimas de Colombia. Puede ser consultada en: https://cifras.unidadvictimas.gov.co/Home/General.

${ }^{2}$ La ARN reporta y actualiza permanentemente las cifras de la población en proceso de reintegración y reincorporación en Colombia. Las cifras pueden ser consultadas en http://www.reincorporacion.gov.co/es/agencia/Paginas/ARN-en-cifras.aspx.
}

Estudios de la Paz y el Conflicto, Revista Latinoamericana, IUDPAS-UNAH, Número Especial 1, 2019, pp. 53-65. DOI: 10.5377/rlpc.v0i0.9503 
Ahora bien, algunas coordenadas epistémicas se hacen necesarias para ubicar desde dónde están construidas estas reflexiones. La primera tiene que ver con que el sujeto y su experiencia son el punto de partida de cualquier proceso de conocimiento, ya que conocer no es otra cosa que poner en relación, de manera que todo aquello que se piensa y se hace es realizado por alguien que tiene voz, que tiene vida y que tiene, por tanto, un punto de ubicación específico en la historia. De ahí que, en los procesos de educación, más que la transmisión de información y de contenidos, se privilegie la producción de conocimiento, de los sujetos y del mundo en el que habitan y por el cual son interpelados esos sujetos.

La segunda está relacionada con la historicidad, la cual hace referencia a que dicha ubicación específica de los sujetos corresponde a sus trayectorias vitales, lo cual le confiere singularidad a su propia historia personal, apartándose de la noción de individualidad ya que incorpora necesariamente la experiencia social de la comunidad y la época en donde ha desarrollado tal trayectoria. Esta clave implica el reconocimiento de los sujetos en su carácter histórico y, por tanto, implicará para cualquier tipo de acción desde la psicología, una pregunta por quiénes son los sujetos con los que trabaja y cómo han llegado a ser lo que son. Como será desarrollado más adelante, este elemento dialoga muy fuertemente con la dimensión de la memoria como construcción de sentido de la experiencia vivida.

Por su parte, la tercera clave está relacionada con la totalidad como lógica de articulación, la cual se configura como una demanda propia de cualquier proceso de producción de conocimiento, pues implica pensar en relación y en clave de las múltiples articulaciones posibles en la aproximación a cualquier fenómeno estudiado; y, finalmente, la dialéctica como forma de razonamiento, que puede recuperarse en por lo menos tres perspectivas alrededor de la idea de movimiento: la dialéctica del sujeto, que se interesa por comprender la doble transformación del sujeto y su mundo en la medida en que están en una relación de co-determinación e interdependencia; la dialéctica como apropiación de la realidad y su movimiento, lo cual implica en el orden del pensamiento reconocer que se requiere más que un cuerpo conceptual o teórico para comprender la realidad, que es siempre cambiante y específica al contexto de problematización que se desarrolle; y la dialéctica como negatividad, en cuanto a que implica la exploración de lo que aún no está construido ni dado, sino de lo que existe como posibilidad o potencialidad.

Este último elemento resulta fundamental en cuanto porta un componente ético particular para la producción de conocimiento, que tiene que ver con el imperativo de ir más allá de las descripciones, y de "lo que hay", para buscar alternativas y opciones de futuro. Este es uno de los elementos que podría distinguir la tradición conocida como "violentología"3, de los esfuerzos y múltiples iniciativas de construcción de paz hoy en Colombia.

Siguiendo entonces esta matriz epistémica, se proponen algunas reflexiones sobre la psicología como disciplina en Colombia, en las que los procesos de formación universitaria y desarrollo profesional no deben estar separados, sino que justamente deben nutrirse permanentemente.

\footnotetext{
${ }^{3}$ El nombre coincide con un libro de reportería gráfica del conflicto armado en Colombia de Stephen Ferry, que siguiendo la "escuela de sociología de los violentologos" decide adoptar su nombre para retratar la complejidad del conflicto más allá de una guerra entre las fuerzas públicas del estado colombiano y los productores de droga. Dicha escuela se desarrolla principalmente entre sociólogos e historiadores que buscan comprender la violencia política, en medio de la negación total de un conflicto armado en Colombia hasta bien entrado el siglo XXI, por lo que constituyó un valiosísimo aporte al pensamiento durante los años más oscuros del conflicto en Colombia.
}

Estudios de la Paz y el Conflicto, Revista Latinoamericana, IUDPAS-UNAH, Número Especial 1, 2019, pp. 53-65. DOI: 10.5377/rlpc.v0i0.9503 


\section{DOS ÓRDENES DE ENTRADA Y SALIDA: LAS TENSIONES DEL SER FORMADO/ LAS TENSIONES DEL QUEHACER DE LA PSICOLOGÍA EN CAMPO}

De acuerdo con los datos del Observatorio de la Calidad en la Educación Superior en Psicología en Colombia, en nuestro país existen 138 programas de psicología, de los cuales 43 han sido reconocidos con registro de alta calidad ${ }^{4}$. Por su parte, de acuerdo con Cornejo (2018), el Sistema Nacional de Información en Educación Superior de Colombia registró en 2016 un total de 875 graduados de psicología de los programas de universidades públicas, y 5363 de universidades privadas en todo el país ${ }^{5}$. A pesar de que la tendencia en matriculas e inscripciones universitarias en los programas de ciencias sociales y humanas tiende a la disminución de estudiantes, la psicología es hoy una de las carreras más populares, y de hecho parece mantenerse en crecimiento permanente.

De entrada, dicha popularidad despierta varias inquietudes: ¿Cómo es percibido el oficio del psicólogo en un país como Colombia? Si la formación universitaria es cada vez más reducida a la formación para el trabajo ¿qué lugar ocupa la expectativa de lucro al escoger una carrera como la psicología en un país como Colombia? Estas son apenas algunas inquietudes sobre el papel. Sobre la realidad de la población que ingresa cada semestre a nuestro programa hay que señalar varias cosas que llaman la atención: las edades de ingreso de los estudiantes son cada vez menores y están alrededor de los 16 años. Tratándose de una institución privada, para muchos de los estudiantes, ingresar en la universidad siempre fue parte del plan, por lo que se asume como algo dado, configurando un elemento de tensión para los docentes que constantemente reclaman el interés y la sensibilidad de los estudiantes frente a los escenarios que se van vinculando en los contenidos curriculares, como retomaré más adelante.

Pero quizás lo que resulta más preocupante, es que los aspirantes a estudiar psicología algunas veces son jóvenes que buscan esforzarse al mínimo, o que piensan que se trata de una disciplina fácil porque tiene que ver con la gente y no con números, lo que les da la impresión de un menor grado de dificultad en comparación con una ingeniería, porejemplo. Por fortuna, existen también estudiantes interesados en forjar un camino de cercanía con las realidades de nuestro país desde la psicología, y en últimas son estos los que logran mantenerse hasta obtener el título.

Más allá de esto, quiero apuntar a discusiones más profundas, no porque esto no sea importante, sino porque incluso en muchos procesos, los estudiantes son capaces de construir caminos que van convirtiéndolos en profesionales comprometidos, aun cuando llegaran sin ninguna pista o deseo de lo que podía implicar su formación universitaria. Quizás justamente ese efecto de desubicación del estudiante al ingresar a la vida universitaria es un síntoma más de la manera en que los procesos de formación se han ido insertando y continúan siendo atrapados por las lógicas del desarrollo neoliberal de nuestras economías de mercado.

Hay un elemento que sobresale como demanda en el proceso de formación de psicólogos y que tiene que ver con las competencias técnicas e instrumentales para el ejercicio

\footnotetext{
${ }^{4}$ Consultado el 11 de julio de 2019 en http://observatorio.ascofapsi.org.co/programas-de-pregrado/

5 "In 2016, Colombian National Information System of Higher Education registered a total of 875 psychology graduates from public institutions and 5,363 from private universities nationwide" (Cornejo, 2018: 6).
}

Estudios de la Paz y el Conflicto, Revista Latinoamericana, IUDPAS-UNAH, Número Especial 1, 2019, pp. 53-65. DOI: 10.5377/rlpc.v0i0.9503 
profesional, las cuales son altamente valoradas por el mercado laboral, como la capacidad de utilizar y evaluar pruebas psicométricas en contextos clínicos y organizacionales, así como la capacidad de realizar evaluaciones diagnósticas.

Si bien estos elementos son deseables en un profesional titulado, la diversidad de la disciplina hace muy difícil que un programa universitario pueda proveer de todos los elementos que un profesional puede requerir en su campo laboral. Recordemos que la profesión implica una versatilidad importante, puesto que los psicólogos se insertan en casi cualquier campo de conocimiento, desde el marketing, el turismo y la educación hasta la elaboración de políticas públicas, etc., superando por mucho la visión clásica del psicólogo como un profesional exclusivo del área de la salud ${ }^{6}$.

Aquí hay que considerar un elemento de gran importancia, que tiene que ver con el papel de la producción de conocimiento pertinente a nuestras realidades en la disciplina. Es decir, que tanto lo que hacemos los programas universitarios les permiten a los estudiantes producir conocimiento, o los prepara simplemente para una juiciosa trayectoria de repetición. En este sentido, y reconociendo el origen colonial de las disciplinas sociales y humanas, la producción de conocimiento y la capacidad de pensar críticamente para encontrar alternativas a las complejas realidades que vivimos en Colombia se hacen más que necesarias, diremos, imperativas. Y es que, por una parte, la formación en habilidades técnicas específicas y los vacíos de información se pueden suplir con procesos breves de formación e incluso de procesos auto guiados -vivimos como nunca un tiempo en el que la globalización tecnológica ha puesto a nuestra disposición más información de la que podemos consultar en toda una vida-; y por otra parte, se requiere superar las visiones que ubican a las disciplinas en el centro de la producción de conocimiento, es decir, hoy pensamos que dicha producción debe ser un esfuerzo por la comprensión, intervención y transformación de las realidades sociales de las comunidades con las que trabajamos, y no simplemente esfuerzos por incrementar los corpus teóricos, conceptuales e instrumentales de las disciplinas.

Esto último es deseable, pero no puede ser la prioridad como ha venido siendo, al menos en un país como el nuestro. Y es que resulta impactante e indignante, el silencio prolongado de la psicología frente al dolor de la guerra, el desplazamiento forzado, el reclutamiento de menores, la narcopolítica, la parapolítica, los falsos positivos, la desaparición y asesinato de líderes sociales, entre tantos otros fenómenos propios de la violencia que ha configurado nuestro país. Hoy por fortuna el panorama parece fértil para otras iniciativas de trabajo a lo largo y ancho del país.

La subjetividad como proceso implica todo un espectro interdisciplinar y eso ha venido trayendo también algunas implicaciones para la psicología, pues incluso desde la perspectiva del desarrollo humano se viene planteando que "como psicólogos y psicólogas, sabemos de la enorme pertinencia que tiene hoy en día la discusión sobre las condiciones materiales, sociales, simbólicas en las cuales se despliega el desarrollo humano y social de las personas" (Robledo, 2008: 12).

Esto si bien se ha traducido en la necesidad de incorporar sobre los procesos que configuran a las personas, una visión que los reconozca como situados histórica y contextualmente, y algunas visiones individualistas han ido perdiendo fuerza, éstas están muy lejos de desaparecer y, de hecho, fundamentan una muy buena parte de esos 138

\footnotetext{
${ }^{6}$ No obstante, ésta viene siendo la discusión más importante del gremio en los últimos años en Colombia, a propósito de la implementación de la normatividad que regula el oficio del psicólogo, y de la cual se desprenden mecanismos de control y vigilancia sobre los procesos de formación del psicólogo como profesional de la salud.

Estudios de la Paz y el Conflicto, Revista Latinoamericana, IUDPAS-UNAH, Número Especial 1, 2019, pp. 53-65. DOI: 10.5377/rlpc.v0i0.9503
} 
programas de los que hablamos inicialmente. Pero ¿cuál es la pertinencia de esto para la discusión que se plantea aquí? Si seguimos nuestra propia argumentación, hay que reconocer que en Colombia los profesionales, investigadores y estudiantes como sujetos sociales, estamos atravesados por los fenómenos de la violencia que se han vivido en nuestro país, y es ese justamente uno de los elementos centrales para una psicología comprometida con la construcción de paz.

Nos hemos acostumbrado a interponer una distancia con el conflicto armado, a pesar de que convivimos con él de manera cotidiana, hemos desarrollado una capacidad para ubicarlo siempre en la exterioridad, en el campesino, el desplazado, el indígena, la víctima. Siempre con un rostro, pero nunca el nuestro. Y a nosotros ¿qué nos pasó? ¿cómo podemos como sujetos no estar atravesados por este conflicto? ¿pueden otras violencias cotidianas estar relacionadas con ello?

La firma del proceso de paz con las FARC implicó un quiebre para la sociedad colombiana, una suerte de experiencia mediadora para hablar de lo que no se habló durante años, décadas, quizás un poco más. Una suerte de intoxicación lingüística (Romano, 2007) o exceso de lenguaje siguió a ese momento, como una decantación necesaria, como un camino en el que la palabra comenzó a dar sentido a lo que hemos vivido, ayudándonos a poner en orden la experiencia. Hablar nos permite dotar de sentido a lo que nos ha pasado. Quizás eso ayude a entender la exacerbada disputa semántica anexa a dicha intoxicación lingüística que rodeó todo el tema del proceso de paz; no hay que olvidar que pasamos de no hablar del conflicto vivido por décadas, ni siquiera durante las conversaciones de La Habana, a no hablar de nada más de cara al plebiscito que buscaba respaldar los resultados de dicho proceso. El sentido, compañero inseparable de la memoria, es un campo de disputa y como tal es un campo político.

Pensar la memoria como ética (Mélich, 2001) implica reconocer las historias de aquellos que ya no están presentes, como también las vivencias de las generaciones que nos preceden, cuya experiencia ha ayudado a construir el presente que nos toca vivir. Es tratar de comprender el sentido de las acciones de otros humanos que nos antecedieron, para la comprensión de nuestro propio presente. Pero es más que eso. La memoria implica un relato de lo que nos pasó, lo que significó para nosotros haber vivido aquello. Nos ayuda a entender el pasado y a hacer algo con él. Es decir que allí donde hay silencio como memoria hay un intento por dejar el pasado atrás, para que no regrese, para que no dialogue con el hoy. Pero requerimos dialogar con él hoy. ¿Qué es lo que nos ha pasado? ¿Dónde nos ubicamos frente asta realidad como sujetos de conocimiento? ¿Cómo estas peguntas repercuten en la formación y el quehacer de los psicólogos?

En relación con el proceso pedagógico, Yuri Arias (2015) propone que es justamente la memoria un agente educativo. Y es que la posibilidad de salir de los relatos de exterioridad para avanzar sobre la comprensión de nuestras propias historias y cómo estas han estado atravesadas por las dinámicas del conflicto, resulta un dispositivo muy potente para construir las bases de la reconciliación. Y es que ¿cómo hablar de paz, sin haber hablado del conflicto? O como propone Estela Quintar: habría que preguntarse también ¿por qué no estamos en paz? Las narraciones múltiples de quienes nos precedieron, pero también de nuestros pares, ayudan en la labor de dotar de sentido ese pasado compartido, y más aún el tiempo por venir. Quizás uno de los daños más importantes del último tiempo en Colombia ha sido el reinado de una narrativa que explicó el conflicto, reduciendo dramáticamente su complejidad, y que heredamos del gobierno de Álvaro Uribe Vélez. En medio de un sinsentido supremamente 
prolongado y de una experiencia desorganizada e incesante de violencia, una historia de buenos y malos arrasó el espacio discursivo colombiano. El tema se hizo contundente: una gente mala -narcoterrorista para ser más precisos-, culpable de todos los males del país, había venido atentando sistemáticamente contra una gente de bien, trabajadora y honrada. Había que acabar con ellos militarmente. Así hubiera que disfrazar gente de guerrilleros para subir el ánimo a la tropa, y por ahí derecho, a un país ansioso de recorrer sus carreteras. Así de simple, el que no estaba de acuerdo con esa narrativa probablemente estaría del lado de los malos. Y un poco así llegamos a una buena parte de los discursos que hoy circulan y que no se cansan de atentar contra el proceso de paz, y contra la justicia transicional.

Un profesional en psicología en Colombia muy seguramente trabajará en algún momento con personas que han sufrido hechos victimizantes, o que los han producido. ¿Cómo mantenerse al margen cuando nuestras propias familias han sido afectadas directa e indirectamente durante tanto tiempo? Justamente, la introducción de la memoria como agente educativo en mi práctica docente ha traído resultados muy potentes. El tránsito del "yo no he vivido la guerra", al reconocimiento del miedo, el dolor y la desesperanza en las configuraciones familiares resulta un detonante para la falta de interés de la que hablaba unas líneas más arriba. En este sentido, la convergencia con otros trabajos de memoria como el que desarrolla la metodología de la memoria encriptada (Dussan, 2017) han sido centrales ${ }^{7}$. El trabajo con narraciones autobiográficas como dispositivos pedagógicos colectivos junto a las salidas de campo han abierto un camino en el que la exploración de la propia historia de los sujetos conecta con ese ámbito político de la memoria en el que ya no se trata de hablar del pasado sino de una apuesta ética y común por el futuro. La posibilidad de escuchar al otro haciendo el mismo ejercicio ha resultado revelador. El espejeo, la proyección, el decantar sentidos en comunidad es la primordial función del ejercicio colectivo. Lo que se dice y lo que se calla se hacen igualmente importantes, y es en virtud de todo esto que se hace posible develar la tensión entre lo que se dice, lo que se hace y lo que se piensa que se hace, porque allí están los otros con quienes se comparte un conocimiento experiencial y vivencial que se mueve a través de cada uno de los participantes.

Estos ejercicios nos han conducido a la pregunta por los saberes de la guerra (Sánchez, 2017) y a descubrir que el conflicto está tan inserto dentro de nuestra subjetividad que no podemos recordar cuándo aprendemos sobre el mismo, sino que aparentemente siempre ha estado allí. El primer efecto de este ejercicio es una suerte de indignación por no saber, un reclamo en clave de incomprensión de la propia ubicación frente a lo que ha sido la realidad del país, diríamos un efecto afectivo y cognitivo al mismo tiempo. Por fortuna, ese reclamo tiene la posibilidad de ser trabajado hacia el compromiso con el quehacer profesional, en últimas, el psicólogo en el lugar desde donde esté está en búsqueda del bienestar, la autonomía y el cuidado del otro.

La exploración de la propia biografía está orientada hacia la operación crítica del yo narrativo, explorando así aquella configuración identitaria que está en movimiento en cada

\footnotetext{
${ }_{7}^{7}$ De hecho, los estudiantes de la Universidad de la Amazonía a través del semillero Inti-Wayra, han tenido un papel central en estas reflexiones. Esto ha sido posible en las salidas de campo y otras actividades de intercambio académico en las que se viene colaborando además con docentes y estudiantes de otras universidades. Actualmente están en proceso de publicación los resultados de una investigación conjunta en la que la apuesta metodológica fue justamente a través de los relatos biográficos orientados por la memoria encriptada de los estudiantes de ambas universidades y sus implicaciones para la construcción de políticas públicas desde abajo en el marco de la reconciliación y los procesos de justicia transicional en Colombia.
}

Estudios de la Paz y el Conflicto, Revista Latinoamericana, IUDPAS-UNAH, Número Especial 1, 2019, pp. 53-65. DOI: 10.5377/rlpc.v0i0.9503 
sujeto, y que incesantemente se transforma en la medida en que el narrador y protagonista sigue viviendo. Por ello hablamos de la centralidad de la vida en esta narración, y en ese sentido, en la centralidad de la experiencia y de la vida para los procesos de producción de conocimiento. De allí que la exploración autobiográfica contribuya al descubrimiento, que es a la vez construcción, del lugar de enunciación como base de la actitud investigativa. De acuerdo con Robledo (2008: 15):

"La práctica de la indagación se podrá constituir, a su vez, en base para los procesos de formación en investigación. Partiendo de las preguntas formuladas por los y las estudiantes, que en algunas ocasiones emergen de sus vivencias, de sus intuiciones, de sus convicciones, de sus primeros conceptos, podremos, gradualmente, transitar hacia las preguntas del discurso duro de nuestra disciplina”.

\section{TODA PSICOLOGÍA ES POLÍTICA}

Se propone la educación universitaria y la formación de sujetos como escenarios ético políticos, justamente porque allí se está gestando también el tipo de vínculo que necesitamos como sociedad. Colombia, caracterizada por este proceso de violencia imparable, requiere de una renovación en las formas en cómo reconstruimos ese tejido social que se ha venido descomponiendo en las últimas décadas. Es en estas formas justamente que puede construirse una vía en la cual el vínculo social sea el valor fundamental a recrear desde lo micro-lógico, para narrar una historia diferente apelando a memorias diversas.

Sería entonces, lamentable por decir lo menos, mantener la reproducción de cierta asepsia disciplinar que se rehúsa a poner el cuerpo, pero que sobre todo arguye no poder sentar posición en virtud de mantener la objetividad que en otro tiempo persiguieron las disciplinas sociales. La pregunta es si estaremos a la altura como para ubicar la dignidad en vez de la cientificidad y la neutralidad en el centro de las preocupaciones y los desafíos para la formación de profesionales en psicología. De acuerdo con Obando (2016) es esto justamente lo que ha permitido a los estudiantes de psicología en Colombia atravesar sus procesos de formación sin necesariamente problematizar lo que implica en sí la construcción de un sujeto político, y en últimas cualquier relación entre psicología y política.

Hablar de la memoria como ética interpela el para qué de la educación y de la formación de sujetos y se sustenta en el reconocimiento del otro como un otro legítimo, en su singularidad, además de mi propia relación con ese otro, una relación entre distintas tramas existenciales luchando por su legitimidad en el mundo. Desde esta perspectiva, es justamente esa lucha por la legitimidad de cada singularidad humana la que cuestiona a los procesos educativos que privilegian la transmisión de información sobre la formación de sujetos. Requerimos hacer posible el surgimiento de sujetos políticos, para romper con la transparencia del significado, con el "esto siempre ha sido así", y con el "eso no tiene que ver nada conmigo".

Es por esto que, en relación con la noción de subjetividad política, vale la pena insistir en que es gracias a la recuperación de la historicidad y las trayectorias vitales de los sujetos que la memoria deviene un instrumento de potenciación.

"Desarrollar un conocimiento situado implica develar, en el marco de las prácticas a través de las cuales nos constituimos como sujetos, las implicaciones que tiene en la construcción de la subjetividad, las condiciones históricas y geográficas de lo que significa ser hombres, mujeres, niñas, niños y viejos, en países como los nuestros." (Robledo, 2008: 15). 
Ahora bien, respecto a las tensiones del quehacer de la psicología en el campo del post acuerdo hay varias reflexiones importantes como aprendizajes de los procesos de reintegración y de reincorporación. Hay que decir que quizás uno de los elementos más importantes es mantener la comprensión de los procesos de salud mental mucho más allá del espectro de las patologías individuales pues si bien es cierto que el malestar expresado en síntomas es manifiesto, no solo en el caso de muchas personas en proceso de desmovilización de grupos armados ilegales (Aponte y Zapata, 2018), lo que supone además de atención personalizada, la comprensión amplia de la salud mental para ir acercándonos cada vez más a una visión de las dinámicas territoriales, las condiciones materiales de existencia y a las trayectorias vitales e institucionales de los sujetos que han hecho parte de los grupos armados ilegales en nuestro país (Aponte, Zapata y Agudelo, 2018).

Además, un enfoque integrador en salud mental en Colombia con visión territorial puede permitirnos superar las estrategias sectorizadas como las tenemos hasta ahora, en la atención diferencial para víctimas, para desmovilizados con la Agencia Colombiana para la Reintegración y para las desmovilizaciones colectivas de las FARC, todas por su parte y muchas veces con los profesionales de un lado en contra de los del otro lado. Las epistemes institucionales (Zemelman, 2007) no pueden ser faro del profesional en psicología, por cuanto en vez de pensarse en lógica de bandos, la construcción del lugar de enunciación de permitir ubicarse en clave de singularidad para sí y frente a los demás.

Además, la noción de historicidad nos ha permitido reconocer que en los Procesos de Desarme, Desmovilización y Reintegración (DDR), en vez de desear un "borrón y cuenta nueva" para los desmovilizados, es importante reconocerlos como sujetos históricos y ubicar la búsqueda de autonomía y de procesos de resignificación en el marco de la singularidad de cada persona (Aponte, et al., 2018). Esto implica ampliar incluso la visión de los enfoques diferenciales, pues aunque éstos han permitido grandes avances, también corren el riesgo de homogenizar la experiencia subjetiva de colectivos como "los indígenas", "las mujeres", etc. (Frieri y Agudelo, 2019).

Por otra parte, los aprendizajes conllevan implicaciones profundas para la investigación, no solo desde una psicología política, sino para toda psicología, pues involucra la pertinencia del estudio de la experiencia subjetivada de lo político, así como asumir como principio el hecho de que "las problemáticas sociales y políticas afectan de manera directa e indirecta el desarrollo subjetivo" (Obando, 2016: 26).

Además, la participación de los psicólogos en la elaboración de políticas públicas es un requerimiento si se aspira a un proyecto ciudadano inspirado en una cultura de convivencia y de respeto por las diversas experiencias de los sujetos sociales en Colombia. Y es que el trabajo de campo nos da cuenta de que es importante romper con el estereotipo que tiene el psicólogo como "radar" de anomalías, pues hasta ahora en relación con el proceso de reincorporación de las FARC no ha sido posible abrir un puente hacia la indagación biográfica de lo que ha sido la guerra para los combatientes.

Algunos elementos parecen estar relacionados con esto. La visión que como colectivo tienen las FARC implica dos argumentos centrales para cerrar las puertas a cualquier tipo de trabajo desde la psicología. El primero tiene que ver con la idea de que el malestar psíquico está relacionado con el reclutamiento forzado, de manera que una y otra vez se insiste en que no se necesita a ningún psicólogo porque en el grupo todo el mundo está por su propia voluntad; pero, además, que no hay ningún loco en las filas de los combatientes. 
El desafío de los años por venir en este sentido será permitir la exploración de las maneras cómo hemos sido afectados por el conflicto, de un lado y de otro, avanzando hacia el reconocimiento de lo que nos une y no tanto de lo que nos distancia, argumento preferido de quiénes promueven la desestructuración de lo poco que se ha logrado con el proceso de paz hasta ahora en Colombia.

Dos elementos más resultan importantes en la reflexión sobre el quehacer de la psicología en tiempos de post acuerdo de paz en nuestro país. El primero ha sido abordado por el investigador Juan Pablo Aranguren quien hace varios años viene trabajando en el proyecto "La ética de la escucha", y el cual se ha planteado la pregunta por el boom de los relatos y la elaboración de narrativas sobre el conflicto en Colombia, pero desde la problematización de quien escucha y su lugar frete al dolor de los demás. Si bien, el proyecto explora otros oficios que también se ven involucrados en la escucha de testimonios de dolor, lo que quisiera plantear aquí es el preocupante escenario laboral de los psicólogos quienes no solamente deben cargar con el peso de las personas a las que acompañan, sino que también muchas veces de sus propios equipos de trabajo en medio de una marcada precariedad laboral.

$\mathrm{Y}$ es que la generalidad es encontrar personas muy cualificadas y con vasta experiencia, constantemente desempleadas o en contratos que muchas veces no superan los tres o cuatro meses. Estas son las condiciones laborales de quiénes se encargan de la reconstrucción de buena parte del tejido social de nuestra sociedad. Es un buen momento para ampliar la mirada sobre lo que se conoce como cuidado al cuidador, incluyendo y problematizando el elemento de la estabilidad laboral para estos profesionales.

El segundo elemento tiene que ver con el imperativo de una postura crítica del gremio y de los propios psicólogos frente a su oficio. Y es que no pocas veces termina realizándose un trabajo que es instrumental a las mismas estructuras de desigualdad y hasta de crimen organizado. Es decir, hay que abrir las preguntas acerca de para qué realizar trabajos de duelo o de reparaciones simbólicas en lugares donde no es posible acompañar los reclamos de justicia de las comunidades. Esto ya se hace manifiesto en algunos territorios en donde la proclama ha llegado a ser: no más memoria, queremos justicia.

Esto de ninguna manera quiere decir que deban abandonarse los procesos de acompañamiento comunitario, sino que los acompañamientos deben hacerse desde el criterio crítico de profesionales que no solo están disponibles una vez que el daño se ha producido, sino que hacen de la búsqueda de la dignidad y la defensa de la vida el centro de sus relaciones y vínculos con las poblaciones, instituciones y organizaciones con las que trabajan en campo. De ahí que la producción de conocimiento sea el hilo de sentido de esta reflexión que ha atravesado la discusión sobre una psicología que indaga y problematiza la realidad de su hacer en el mundo. Asumir una postura frente a los desafíos del país pasa también por estar al tanto de nuestra intervención en las dinámicas sociales y su impacto en la vida de las comunidades, "adquirimos un compromiso ético y político, el cual implica formarnos para contribuir a un proyecto de país y de mundo, en el cual puedan materializarse los dos principios fundamentales para tener una vida digna: la igualdad y la libertad. Éste es el reto que enfrentamos hoy cuando acompañamos la educación de cientos de estudiantes en nuestros programas de psicología" (Robledo, 2008: 16).

La pedagogía recoge en su intencionalidad formativa relaciones que se construyen con base en horizontes éticos de reconocimiento (Fernández, Orozco y Ospina, 2019), en tanto que producir conocimiento implica entonces asumir una postura epistémica, comprometiéndose con el mundo de la vida en perspectiva crítico-hermenéutica; un acto que 
busca permitirnos entender las cosas que vivimos en nuestra vida cotidiana, es un conocimiento del mundo y de la realidad social en la que vivimos insertos.

\section{REFERENCIAS}

Agencia para la Reincorporación y Normalización ARN Colombia (2019) ARN en cifras corte diciembre 2019. Disponible en: http://www.reincorporacion.gov.co/es/agencia/Paginas/ARN-en-cifras.aspx. Consultado el 18 de febrero de 2020.

Aponte, DM., Durán, A., Laverde, D., Zapata, JG., Agudelo, DM., Gaitán, D., y Granados, A. (2018). Encuentros humanizantes: implementación de una propuesta, en perspectiva de salud mental, para la atención de personas desmovilizadas de grupos armados en proceso de reintegración, sus familias y comunidades. Bogotá: Universidad Externado de Colombia.

Aponte, DM., Zapata, JG., y Agudelo, DM. (2018). "Mental Health, Trajectories and Quality of Life: A Proposal for a New Understanding of DDR Processes in Colombia". En Atieno C., Robinson, C. (Eds.), Post-conflict Security, Peace and Development. Perspectives from Africa, Latin America, Europe and New Zealand. Springer Briefs in Environment, Security, Development and Peace (Vol. 13). Berlin: Springer International Publishing.

Aponte, DM., y Zapata, JG. (2018). Salud mental en el contexto DDR: trayectorias, espectros de sufrimiento y calidad de vida en población desmovilizada del conflicto armado colombiano. Bogotá: Universidad Externado de Colombia. Facultad de Ciencias Sociales y Humanas. Centro de Investigaciones sobre Dinámica Social; Agencia para la Reincorporación y la Normalización (ARN). (Disponible online: http://search.ebscohost.com/login.aspx?direct=true\&db=cat05988a\&AN=uec. 255877\&1 ang $=\mathrm{es} \&$ site $=\mathrm{eds}$-live $\&$ scope $=$ site $)$.

Arias, Y. (2015). La memoria como agente educativo. Bogotá: Aniwaki.

Cornejo, W. (2018). "The Teaching of Psychology in Colombia: The Challenges for the Discipline and High Quality", International Journal of Psychological Research, 11(1), 68 .

Dussán, D. (2017). El poder del miedo en la reconfiguración de la Utopía. Tesis de grado no publicada para obtener el título de PhD en Conocimiento y Cultura en América Latina. México: Instituto de Pensamiento y Cultura en América Latina (IPECAL).

Fernández, OL., Orozco, M., y Ospina, DA. (2019). "Educación, democracia, construcción de paz y memoria social. Aproximaciones al estado del arte en contextos de conflicto armado en Colombia". En: Saberes, conocimientos y epistemes de la investigación en América Latina: algunos temas relevantes. Manizales: Centro Editorial Universidad Católica de Manizales.

Ferry, S. (2012) Violentología. Un manual del conflicto colombiano. Bogotá: Icono editorial. Frieri, S., y Agudelo, D.M. (2019). "Memoria, saberes e intersubjetividades en el aula: prácticas culturales para la restauración de vínculos sociales". En: Gutierrez, M., Olarte Á. (Eds.), Cátedra Unesco. Derechos humanos y violencia: gobierno y gobernanza. Desplazamiento forzado, análisis para la prevención y la restauración de los vínculos sociales rotos. Bogotá: Universidad Externado de Colombia.

Gobierno de Colombia y Fuerzas Armadas Revolucionarias de Colombia - Ejército del Pueblo (FARC-EP). Acuerdo General para la Terminación del Conflicto y la

Estudios de la Paz y el Conflicto, Revista Latinoamericana, IUDPAS-UNAH, Número Especial 1, 2019, pp. 53-65. DOI: 10.5377/rlpc.v0i0.9503 
Construcción de una Paz Estable y Duradera, de 24 de noviembre de 2016. Bogotá: Alto Comisionado para la Paz.

Mèlich, JC. (2001). La Ausencia de Testimonio. Ética y pedagogía en los relatos del holocausto. Barcelona: Anthropos.

Obando, O. (2016). Psicología política sobre la participación de jóvenes desvinculados y desmovilizados del conflicto armado colombiano (Vol. 1: Perspectivas críticas en psicología política latinoamericana). Cali: Programa Editorial Universidad del Valle.

Robledo, Á. (2008). "La formación de psicólogos en Colombia", Universitas Psychologica, 7(1), 9-18.

Romano, V. (2007). La Intoxicación lingüística. El uso perverso de la lengua. Barcelona: El Viejo Topo.

Sánchez, A. (2017). Los saberes de la guerra. Memoria y conocimiento intergeneracional del conflicto en Colombia. Bogotá: Siglo del Hombre Editores y Universidad Nacional de Colombia.

Zemelman (2007). El ángel de la historia: Determinación y autonomía de la condición humana. Barcelona: Anthropos.

Zemelman, H. (2011). De la historia a la política. La experiencia de América Latina. La Paz: Colectivo Grito del Sujeto.

\section{REFERENCIA DE LA AUTORA}

Diana Marcela Agudelo Ortiz, Psicóloga. Maestra en Estudios de la Cultura con mención en Políticas Culturales. Candidata a PhD en Conocimiento y Cultura en América Latina. Docente investigadora del programa de Psicología, el Laboratorio Interdisciplinar de Ciencias y Procesos Humanos (LINCIPH), y del grupo de investigación Salud, conocimiento médico y sociedad de la Facultad de Ciencias Sociales y Humanas, Universidad Externado de Colombia. diana.agudelo@uexternado.edu.co. ORCID: https://orcid.org/0000-00020729-5708

Recibido: 23-01-2020

Aceptado: 04-02-2020

(c) (1) Licencia Creative Commons Reconocimiento 4.0 (CC BY 4.0).

Estudios de la Paz y el Conflicto, Revista Latinoamericana, IUDPAS-UNAH, Número Especial 1, 2019, pp. 53-65. DOI: 10.5377/rlpc.v0i0.9503 